\title{
On Wallis' formula
}

\section{By Donat K. Kazarinoff.}

In the course of mathematical progress new truths are discovered while older ones are sometimes more precisely articulated and often generalised. Because of their elegance and simplicity, however, some classical statements have been left unchanged. As an example, I have in mind the celebrated formula of John Wallis,

$$
\frac{1}{\sqrt{ }(\pi n)}>\frac{1.3 .5 \ldots(2 n-1)}{2.4 .6 \ldots(2 n)}>\frac{1}{\sqrt{ }\left\{\pi\left(n+\frac{1}{2}\right)\right\}}, n=1,2, \ldots,
$$

which for more than a century has been quoted by writers of textbooks. Usually this formula is written as

$$
\frac{1.3 .5 \ldots(2 n-1)}{2.4 .6 \ldots(2 n)}=\frac{1}{\sqrt{ }\{\pi(n+\theta)\}}, 0<\theta<\frac{1}{2}, n=1,2, \ldots .
$$

In this note it is shown that $\frac{1}{4}<\theta<\frac{1}{2}$. Unquestionably, inequalities similar to this one can be improved indefinitely but at a sacrifice of simplicity, which is why they have survived so long.

The proof of my assertion that $\frac{1}{4}<\theta<\frac{1}{2}$ is based upon a property of the function

$$
\Phi(a)=\int_{0}^{\pi / 2} \sin ^{\alpha} x d x=\frac{\sqrt{\pi}}{2} \frac{\Gamma\left(\frac{a+1}{2}\right)}{\Gamma\left(\frac{a+2}{2}\right)},-1<\alpha<\infty .
$$

Upon logarithmic differentiation of $\Phi(a)$, we have

$$
D_{\alpha} \log \Phi(\alpha)=\frac{1}{2}\left\{\frac{\Gamma^{\prime}\left(\begin{array}{c}
a+1 \\
2
\end{array}\right)}{\Gamma\left(\begin{array}{c}
a+1 \\
2
\end{array}\right)}-\frac{\Gamma^{\prime}\left(\frac{a+2}{2}\right)}{\Gamma\left(\frac{a+2}{2}\right)}\right\} .
$$

Making use of Legendre's formula that ${ }^{1}$

$$
\frac{\Gamma^{\prime}(\beta)}{\Gamma(\beta)}=-C+\int_{0}^{1} \frac{t^{\beta-1}-1}{t-1} d t, 0<\beta<\infty,
$$

$C$ being Euler's constant, we can write

$$
D_{a} \log \Phi(a)=-\int_{0}^{1} \frac{t^{a} d t}{1+t} \text { and } D_{a}^{2} \log \Phi(a)=-\int_{0}^{1} \frac{t^{a} \log t}{1+t} d t
$$

1 E. T. Whittaker and G. N. Watson, Modern Analysis 4th ed. (Cambridge, 1927), p. 260. 
The property of $\Phi(a)$ fundamental to our discussion is that for $-1<\alpha<\infty$,

$$
D_{a}^{2} \log \Phi(a)-\left\{D_{a} \log \Phi(a)\right\}^{2}>0 .
$$

This is, of course, equivalent to the statement that the reciprocal of $\Phi(\alpha)$ has an everywhere negative second derivative. Geometrically this signifies that the harmonic mean of any two ordinates of the graph of $\Phi(a)$ is greater than the ordinate equidistant from them.

To prove $\left(^{*}\right)$ we estimate the integrals

$$
I=\int_{0}^{1} \frac{t^{a}}{1+t} d t \text { and } J=-\int_{0}^{1} \frac{t^{a} \log t}{1+t} d t .
$$

We now subdivide the interval $-1<a<\infty$ into two intervals, $-1<a<0$ and $0 \leqslant a<\infty$. In the interval $0 \leqslant a<\infty$,

$$
I \leqslant \frac{\log 2}{1+a} \text { and } J>\frac{1}{2(1+a)^{2}} .
$$

The first estimate may be obtained by integrating $(a+1) I$ by parts and thereby noting that $(a+1) I$ is a monotone decreasing function of $a$ having the value $\log 2$ for $a=0$. The second estimate is obtained from the inequality

$$
J>-\frac{1}{2} \int_{0}^{1} t^{a} \log t d t
$$

We may now conclude that

$$
J-I^{2}>\frac{1}{2(1+\alpha)^{2}}-\left(\frac{\log 2}{1+\alpha}\right)^{2}>0,0 \leqslant a<\infty .
$$

On the interval $-1<a<0$, we have the more precise estimates

Hence,

$$
I<\frac{1}{1+a}-\frac{1}{2(2+a)} \text { and } J>\frac{1}{(1+a)^{2}}-\frac{1}{2\left(a+\frac{3}{2}\right)^{2}} .
$$

$$
J-I^{2}>\frac{1}{(2+a)(1+a)}-\frac{1}{4(2+a)^{2}}-\frac{1}{2\left(a+\frac{3}{2}\right)^{2}} .
$$

But $(2+a)(1+\alpha)<\left(a+\frac{3}{2}\right)^{2}$; and consequently

$$
J-I^{2}<\frac{2}{4\left(\alpha+\frac{3}{2}\right)^{2}}-\frac{1}{4(2+\alpha)^{2}} .
$$

Replacing 3 by 2 , we have

$$
J-I^{2}>\frac{1}{4(2+a)^{2}},-1<a<0 .
$$

Finally by $(a)$ and $(b), J-I^{2}>0,-1<a<\infty$, which establishes $\left({ }^{*}\right)$. 
For any positive $a$ we now consider $\Phi(a), \Phi(a-1)$, and $\Phi(a+1)$. By $\left({ }^{*}\right), \Phi(a)$ is less than the harmonic mean of $\Phi(a-1)$ and $\Phi(a+1)$; and therefore $\Phi(\alpha)$ is less than the geometric mean of $\Phi(a)$ and the harmonic mean of $\Phi(\alpha-1)$ and $\Phi(\alpha+1)$. This leads to the fact that

$$
\frac{\Gamma\left(\frac{a+1}{2}\right)}{\Gamma\left(\frac{a+2}{2}\right)}<\frac{2}{\sqrt{ }(2 a+1)} .
$$

As a subcase of this result, we have found that

$$
\frac{1.3 .5 \ldots(2 n-1)}{2.4 .6 \ldots(2 n)}<\frac{1}{\sqrt{ }\left\{\pi\left(n+\frac{1}{4}\right)\right\}},
$$

which was the inequality to be established.

The University of Michigan, Ann Arbor, Michigan, U.S.A: 